\title{
Dynamics of Spreading of Small Droplets of Chainlike Molecules on Surfaces
}

\author{
J. A. Nieminen ${ }^{1}$ and T. Ala-Nissila ${ }^{1,2}$ \\ ${ }^{1}$ Department of Physics \\ Tampere University of Technology \\ P.O.Box 692 \\ FIN - 33101 Tampere \\ Finland \\ ${ }^{2}$ Research Institute for Theoretical Physics \\ University of Helsinki \\ P.O. Box 9 (Siltavuorenpenger $20 \mathrm{C}$ ) \\ FIN - 00014 University of Helsinki \\ Finland
}

January 3, 1994 


\begin{abstract}
Dynamics of spreading of small droplets on surfaces has been studied by the molecular dynamics method. Simulations have been performed for mixtures of solvent and dimer, and solvent and tetramer droplets. For solvent particles and dimers, layering occurs leading to stepped droplet shapes. For tetramers such shapes occur for relatively deep and strong surface potentials only. For wider and more shallow potentials, more rapid spreading and rounded droplet shapes occur. These results are in accordance with experimental data on small non - volatile polymer droplets.

PACS numbers: 68.10Gw, 05.70.Ln, 61.20.Ja, 68.45Gd
\end{abstract}


Experiments on the dynamics of spreading of tiny non - volatile liquid droplets have revealed a variety of droplet shapes on the molecular scale [1, 2, 3, 4] which cannot be explained by continuum hydrodynamic theories $[5,6,0$, 8]. These experiments have created a need for more microscopic models [8]. Some analytic models have been successful in describing dynamical layering 9, 10, 11. To obtain more microscopic understanding, molecular dynamics simulations have been performed [12, 13]. In particular, Nieminen et al. [14] simulated Lennard - Jones (LJ) particles and observed the appearance of a precursor film, dynamical layering, and a crossover from almost linear $\left(\sim t^{0.9}\right)$ to $t^{0.5}$ behavior for the width of the precursor [7, 8].

However, recent experiments using a variety of polymer liquids on energetically different surfaces show features which are beyond the simple LJ model of a liquid [1, 2, 3, 4]. For example, a droplet of squalane develops only a tiny foot preceding the droplet [2], whereas in the case of polydimethylsiloxane (PDMS) a monolayer thick precursor layer is seen [2], except on silver where the droplet has a spherical cap and Gaussian tails at late times [3]. Furthermore, tetrakis (2 - ethylhexoxy) - silane develops multiple (up to four) precursor layers and a tiny cap in the center of the droplet [1]. Venäläinen et al. [15 have recently demonstrated that most droplet shapes can be qualitatively reproduced by an effective solid on solid model where interparticle and substrate interactions are varied. However, there still remains an obvious need to understand the role of microscopic solid - liquid and liquid - liquid interactions, temperature, and, in particular, the chainlike morphology of the molecules. Although most experiments on tiny droplets have been done with rather short molecules beneath the static entanglement regime [16], re- 
cent work shows [3] how spherical droplet shapes can occur due to strong blocking effects between short chainlike molecules. This is the motivation for the present work, where we present a study where the chainlike structure of the spreading liquid and the influence of the surface potential are taken into account microscopically within the molecular dynamics (MD) method.

We consider a model system of a mixture of "solvent" (single atom) particles and "polymers" (short effective chainlike molecules) which interact with each other via an LJ potential, with potential parameters $\sigma_{f}$ and $\epsilon_{f}$ for the width and depth of the potential, respectively. The substrate is modeled by a flat continuum LJ material [14. For the substrate - fluid interaction, the depth of the potential is comparable to $\epsilon_{s} \sigma_{s}^{3}$ and the width to $\sigma_{s}$ (see Ref. [14]). The $n$ - mers in the system consist of $n$ LJ particles, which are interconnected by a very rigid but orientationally isotropic harmonic oscillator pair potential $V_{c}=\frac{1}{2} k\left(r-r_{0}\right)^{2}$, where $k=100 \epsilon_{f} / \sigma_{f}^{2}$. There is also an angle dependent potential $V_{\theta}=\epsilon_{\theta}(\cos \theta+1)$, which favors linear molecules. Here we present results for $\epsilon_{\theta}=10 \epsilon_{f}$ which turns out to be the most interesting case; results for more flexible molecules are similar, and will be published elsewhere [17]. Due to the strong pair potential between subsequent atoms comprising an $n$ - mer, a strong repulsion is created if any $n$ - mers try to spatially overlap.

The dynamics of the system is described by the usual equations of motion [14]: $d r_{i} / d t=p_{i} / m_{i}, d p_{i} / d t=\nabla_{i} V-\eta p_{i}$, and $d \eta / d t=\left[\Sigma_{i}\left(p_{i}^{2} / m_{i}\right)-\right.$ $N k T] / N k T \tau^{2}$, where $N$ is the number of degrees of freedom, $T$ the temperature for the thermostat, and $\tau$ is a relaxation time. The time scales are here chosen in the same way as in Ref. [14]. Initially a ridge - shaped droplet 
is constructed with periodic boundary conditions along the direction of the ridge. The spreading takes place in the direction perpendicular to the ridge. The equations of motion are solved using modified velocity Verlet algorithm (see e.g. [18] and [19]).

The initial configuration is constructed so that the $n$ - mers are bent 90 degrees at each joint, while their directions are arbitrary. To attain an optimum packing density the system is compressed, after which the system is allowed to find a lower energy state. After finding the best possible configuration we switch on the substrate interaction and allow the system to evolve.

In the previous study of Nieminen et al. [14 the temperature was $k T_{s}=$ $0.8 \epsilon_{f}$, which is well above the triple point of an LJ material [20]. In the present case the larger mass and binding energy of the $n$ - mers allows us to systematically vary both the surface potential and the temperature, and thus e.g. study the effects of changing the viscosity of the liquid. In the present work, we employ several sets of substrate interaction parameters: $\epsilon_{s}=5 \epsilon_{f}$ and $\sigma_{s}=\sigma_{f}$ (used in Ref. [14), denoted as $S_{I} ; \epsilon_{s}=\epsilon_{f}$ and $\sigma_{s}=5.0 \sigma_{f}$ $\left(S_{I I}\right) ; \epsilon_{s}=0.02 \epsilon_{f}$ and $\sigma_{s}=7.3 \sigma_{f}\left(S_{I I I}\right) ;$ and $\epsilon_{s}=0.002 \epsilon_{f}$ and $\sigma_{s}=7.3 \sigma_{f}$ $\left(S_{I V}\right)$. The differences between these potentials can be characterized by their steepness (gradient) $\left(\sim \epsilon_{s} \sigma_{s}^{2}\right)$, depth, and width. The potential $S_{I I}$ is much steeper and deeper than $S_{I}$, while $S_{I I I}$ and $S_{I V}$ are both relatively shallow, the latter being particularly flat.

First we describe results for mixtures of solvent and dimer particles. Qualitatively, a dimer concentration of $50 \%$ or $100 \%$ does not alter the essential features of spreading as compared to a pure solvent droplet. Dimer droplets 
exhibit well separated layers such as observed for solvent droplets for $S_{I}$, $S_{I I}$ and $S_{I I I}$. However, in order to clearly observe this, a somewhat deeper substrate potential $S_{I I}$ must be used (for $S_{I}$ only one precursor layer forms). Despite this slight difference dimer droplets show a rather clear crossover from the almost linear to $t^{0.5}$ spreading as described in Ref. [14] for solvent droplets. The presence of dimers, however, makes the spreading dynamics slightly slower.

In the case of tetramers, effects arising from the chain structure of the liquid become more apparent. The first striking feature is a dynamical ordering of the rather stiff chains along the "flow" of the spreading droplet, perpendicular to the surface (Fig. 1). The nature of layer separation is also changed. Only for the very strong surface potential $S_{I I}$ a precursor layer with thickness of about one monolayer is formed. When surface potential becomes more shallow, a distinct change in the droplet shapes occur. For $S_{I I I}$ a vertically continuous precursor layer whose effective thickness is two layers is seen. When the substrate potential is further weakened to $S_{I V}$, the two layers tend to merge together. This leads to a noticeably more rounded droplet configurations than for dimers. The rate of spreading of the droplet also becomes faster from $S_{I I}$ to $S_{I I I}$. This is consistent with the observation that for a strongly attractive, narrow surface potential the tetramers become tightly packed parallel to the surface, which slows down their migration in the effectively two dimensional layer [3]. Such layers also remain relatively well separated. On the other hand, a wider surface potential does not force the tetramers in layers, thus allowing faster dynamics and formation of a more rounded droplet configuration. Mixing of the layers in Fig. 1 is seen 
which leads to a very complex structure within the precursor layer. When the substrate interaction is still weakened from $\left(S_{I I I}\right)$ to $\left(S_{I V}\right)$, no further increase in the rate of spreading is not seen.

We have also studied the effect of increasing tetramer concentration starting from a pure solvent droplet, as shown in Fig. 2. This slows down the spreading rate in analogy with the dimer case. Most importantly, we find that the width of the precursor film $w$ obeys a finite size scaling form as a function of the number of LJ particles $N$ [14, 17] $w=t^{x} \phi\left(t / N^{y}\right)$, where $x \approx 9 / 10$ [7], and $y \approx 0.67 / x$. This was checked for $2380 \leq N \leq 4080$. This indicates that the spreading is not purely diffusive but rather caused by a combination of the pressure of the cap and collective migration of the tetramers [21]. The scaling function $\phi(z) \sim$ const. for $z \ll 1$, and $\phi(z) \sim z^{1 / 2-x}$ for $z \gg 1$. Details of these results will be published elsewhere [17].

Next we study the density profiles of the droplets in order to make comparison with experiments [1, 国, 每, 四. As seen in Fig. 3(a), the density profile for a solvent droplet shows a stepped precursor layer for $S_{I I}$, indicating separation of layers. Results for dimers are analogous, with slightly more rounded step edges for weaker potentials. For tetramers in the case of $S_{I I}$, the density profile shows a stepped shape, indicating dynamical layering. For weaker potentials the comparison of snapshots and profiles shows mixing of the layers. For $S_{I I I}$ the precursor film on the whole is fairly thick and the density continuosly increases towards the centre. The density profile is seen to develop towards a rounded cap [3], which is most clearly seen in Fig. 3(b) for the case of $S_{I V}$. Additional studies with more flexible chains and octamers support these conclusions [17]. 
The profiles of Fig. 3 bear a remarkable qualitative resemblence to some of the experimental ones. The stepped profile of Fig. 3(a) is qualitatively similar to that of a tetrakis droplet consisting of star - shaped molecules [1]. Our results indeed suggest that for spherical or dimer molecules separation of layers tends to occur. On the other hand, the profile of the tetramer droplet (Fig. 3(b)) resembles the profiles of a PDMS droplet on silver [3], and on silicon [2]. These more rounded shapes should occur for chainlike molecules, when the surface attraction is not strong. Furthermore, on silicon a change of the droplet from a rounded towards a stepped shape was observed on a "high - energy" surface. Our results suggest that this indicates increased substrate attraction, which tends to separate the layers. A quantitative comparison of our results with experiments is difficult, however, since experimental droplets are microscopic in the vertical direction only. Nevertheless, our results support the conclusion that weakening the surface potential causes faster spreading and more rounded droplet shapes for polymeric liquids. Faster spreading of the precursor film for weaker potentials has also been predicted in Ref. [9].

Finally, we have also studied the effect of temperature. As expected, increasing the temperature increases the spreading rate. For solvent droplets $\left(S_{I I}\right)$ changing the temperature from $T=T_{S}$ to $T=1.5 T_{S}$ simply makes the steps more rounded. For tetramer droplets in the case of $S_{I I I}$, the effects are relatively small. For $T=4 T_{S}$ the profile shows a minimum at the centre of the droplet, after the vanishing of the cap. This is followed by a fairly flat rounded profile, as seen in Ref. [3].

To summarize, in this work we have studied effects arising from the chain- 
like molecular structure within spreading droplets, and the form of the surface potential. Our model simulations suggest that for relatively strongly attracting surfaces, dynamical layering and stepped droplet shapes tend to occur. On the other hand, the mixing of chainlike molecules opposes this trend leading to more rounded droplet shapes on weaker substrates. Our results also support the conclusion that weaker substrate attraction enhances the rate of spreading. These results are in agreement with experiments [1, 2, 3, 4]. We hope that our work inspires further systematic experiments on the effects of chain lengths and surface potentials on the spreading of tiny droplets.

Acknowledgements: We wish to thank K. Kaski, R. Swendsen, and O. Venäläinen for useful discussions, and S. Herminghaus for a critical reading of the manuscript. This work has been supported by the Academy of Finland. 


\section{Figure Captions}

Fig. 1(a)-(b). Snapshots of a projection of a three dimensional pure tetramer droplet for potential $S_{I V}$, with $N=2380$. Times correspond to 50 and 75 in reduced units [14]. Mixing of the layers is clearly visible. Units in this and the other figures have been obtained from $\sigma_{f}=2.6 \AA$. Due to the effective nature of the chainlike molecules the length scales should be considered effective only.

Fig. 2. The effect of tetramer concentration $c$ on the time evolution of the precursor width $w$ for potential $S_{I I}: c=0$ (full line), $c=0.5$ (dash-dotted line), and $c=1$ (dotted line).

Fig. 3. Smoothed density profiles for two different droplets. (a) A solvent droplet for $S_{I I}$ and (b) a tetramer droplet for $S_{I V}$. The relative times are $t_{1}$, $4 t_{1}$, and $6 t_{1}$. 


\section{References}

[1] F. Heslot, N. Fraysse, and A. M. Cazabat, Nature 338, 640 (1989); F. Heslot, A. M. Cazabat, P. Levinson, and N. Fraysse, Phys. Rev. Lett. 65, 599 (1990).

[2] F. Heslot, A. M. Cazabat, and P. Levinson, Phys. Rev. Lett. 62, 1286 (1989).

[3] U. Albrecht, A. Otto, and P. Leiderer, Phys. Rev. Lett. 68, 3192 (1992).

[4] M. P. Valignat, N. Fraysse, A. M. Cazabat, and F. Heslot, Langmuir 9, 601 (1993); N. Fraysse, M. P. Valignat, A. M. Cazabat, F. Heslot, and P. Levinson, J. Coll. Interface Sci. 158, 27 (1993).

[5] L. H. Tanner, J. Phys. D 12, 1473 (1979).

[6] P. G. de Gennes, Rev. Mod. Phys. 57, 827 (1985).

[7] J. F. Joanny and P. G. de Gennes, J. Physique 47, 121 (1986).

[8] L. Leger and J. F. Joanny, Rep. Prog. Phys. 55, 431 (1992).

[9] D. B. Abraham, P. Collet, J. de Connick, and F. Dunlop, Phys. Rev. Lett. 65, 195 (1990); J. Stat. Phys. 61, 509 (1990).

[10] D. B. Abraham, J. Heiniö, and K. Kaski, J. Phys. A. 24, L309 (1991); J. Heiniö, K. Kaski, and D. B. Abraham, Phys. Rev. B 45, 4409 (1992); E. Cheng and C. Ebner, Phys. Rev. B 45, 12056 (1992).

[11] P. G. deGennes and A. M. Cazabat, C. R. Acad. Sci. 310II, 1601 (1990).

[12] J. Hautman and M. L. Klein, Phys. Rev. Lett. 67, 1763 (1991).

[13] J.-x. Yang, J. Koplik, and J. R. Banavar, Phys. Rev. Lett. 67, 3539 (1991).

[14] J. A. Nieminen, D. B. Abraham, M. Karttunen, and K. Kaski, Phys. Rev. Lett. 69, 124 (1992); J. A. Nieminen, A. Lukkarinen, K. Kaski, and D. B. Abraham, unpublished (1993).

[15] O. Venäläinen, T. Ala-Nissila, and K. Kaski, Europhys. Letters 25, 125 (1994); cond-mat@babbage.sissa.it 9311059.

[16] P.-G. de Gennes, Scaling Concepts in Polymer Physics (Cornell University Press, Ithaca, N.Y. 1979); M. Doi and S. F. Edwards, The Theory of Polymer Dynamics (Oxford University Press, Oxford, 1986). 
[17] J. A. Nieminen and T. Ala - Nissila, University of Helsinki preprint HU - TFT - 93 - 62 (1993).

[18] J. P. Hansen and L. Verlet, Phys. Rev. 184, 151 (1969).

[19] A. P. Sutton, J. B. Pethica, H. Rafii-Tabar, and J. A. Nieminen, in Electron Theory in Alloys Design, eds. D. G. Pettifor and A. H. Cottrell (Institute of Metals, London, 1992).

[20] A. J. C. Ladd and L. V. Woodcock, Mol. Phys. 36, 611 (1978).

[21] Diffusive spreading would lead to $y=1$. 